\title{
Un Análisis del tratamiento didáctico del producto notable (cuadrado de la suma de dos términos) en el libro de texto hipertexto de matemáticas $\mathbf{8}^{1}$
}

\author{
An Analysis of the remarkable product didactic treatment (square \\ of the sum of two terms) in the textbook math hypertext 8
}

\section{Uma Análise do tratamento notável didática produto (quadrado da soma de dois termos) no livro de matemática hipertexto 8}

Recibido: mayo de 2013

Aceptado: agosto de 2013
Diego Guerrero Garay ${ }^{2}$

Ángel Danilo Moreno Prieto ${ }^{3}$

\section{Resumen}

En este trabajo se presentan los resultados de un análisis hecho en el marco de la investigación realizada en el libro de texto hipertexto 8 unidad número cuatro, en la cual se aborda el concepto de productos y cocientes notables, el trabajo se centra en el tipo de tratamiento didáctico que se da en el libro de texto a dicho concepto a través del uso de la teoría de las representaciones semióticas expuestas por (Duval, 2004). El análisis de texto a través de (Piñuel, 2002) y algunos aspectos algebraicos basados en (Kieran, 1989). Finalmente se presentan las conclusiones en torno al análisis realizado.

Palabras clave: Otras nociones de Educación Matemática; sistemas de representación; semiótica; transformación; matemáticas escolares; álgebra; polinomios; producto notable.

\begin{abstract}
In this paper we present the results of an analysis in the framework of the research in the hypertext textbook 8 piece number four, in which he addresses the concept of products and quotients notable work focuses on the type of didactic treatment given in the textbook for the concept through the use of semiotic representations theory expressed by (Duval, 2004). The analysis of text through (Piñuel, 2002) and some algebraic aspects based on (Kieran, 1989). Finally we present our conclusions on the analysis performed.

Keywords: Other notions of Mathematics Education; systems of representation, semiotics processing; school math, algebra, polynomials remarkable product.
\end{abstract}

$1 \quad$ Articulo de Investigación

2 Universidad Distrital Francisco Jose de Caldas, Bogotá, Colombia. Contacto: diegoggaray@hotmail.com

3 Universidad Distrital Francisco Jose de Caldas, Bogotá, Colombia. Contacto: oteirp90@hotmail.com 


\section{Resumo}

Neste artigo apresentamos os resultados de uma análise no âmbito da pesquisa no hipertexto livro de 8 peças número quatro, na qual ele aborda o conceito de produtos e quocientes notável trabalho centra-se no tipo de tratamento didáctico dada no livro de texto para o conceito através da utilização da teoria das representações semiótica expresso por (Duval, 2004). A análise por meio de texto (Piñuel, 2002) e alguns aspectos algébricos baseado em (Kieran, 1989). Por fim, apresentamos nossas conclusões sobre a análise realizada.

Palavras-chave: Outras noções de Educação Matemática, sistemas de representação, de processamento de semiótica, matemática do ensino médio, álgebra, polinômios produto notável.

\section{Presentación del problema}

Teniendo en cuenta lo dicho por Maldonado, Rodríguez y Tuyub (2007) quienes afirman que los libros de texto son considerados los "principales recursos o medios de difusión de los saberes matemáticos", además de ser "medios que de alguna manera norman el tipo de práctica docente" (p.43). Una tensión se centra en que la mayoría de textos usan como herramienta para la interiorización del concepto la mecanización de algoritmos que según (Sánchez y López, 2009). Pueden "causar errores en el aprendizaje cuando el niño acude al uso de éstos mecánicamente, sin considerar si la información analógica es significativa", éste hecho afecta casi todas las ramas de las matemáticas, incluyendo el tratamiento de los productos notables que como (Cervantes, 2010). Afirma, normalmente el profesor de matemáticas recurre a este recurso para explicar reglas fijas lo que conlleva a que el alumno entre en duda sobre la veracidad del procedimiento.

Ahora bien, sabiendo que la mecanización de los procedimientos y la no interiorización del concepto es una de las tensiones, la otra centrará su mirada en la relación que hay entre el libro de texto y la gestión del docente, puesto que en muchas ocasiones como lo menciona (Azcárate y Serrado, 2006). Se convierte en el elemento clave para planificar el desarrollo de los temas a abordar, lo que trae como consecuencia para el docente un desgaste en cuanto a sus teorías didácticas.

\section{Marco conceptual}

En los libros de texto se privilegian el uso de diferentes tipos de representaciones, de acuerdo con lo expuesto por (Duval, 2004). Existen tres tipos de representación semiótica los cuales son: lenguaje, comprendida por enunciados, formulas y teoremas; la visualización en la cual se encuentran los grafos, figuras geométricas y los esquemas; y por último la imagen que se representa por medio de dibujos.

Estos tres tipos de representación pueden sufrir dos tipos de transformación que son: tratamiento y conversión, el primero hace referencia a los cambios que tiene un objeto matemático dentro de un mismo registro de representación, por ejemplo, $6(x+y)$ se puede expresar como $6 x+6 y$, el segundo propone el paso de un registro de representación a otro, ejemplo, $6(\mathrm{x}+\mathrm{y})$ se puede expresar geométricamente como un rectángulo de base $6 \mathrm{y}$ altura $\mathrm{x}+\mathrm{y}$.

Para hablar de los problemas verbales que puede o no presentar el libro de texto nos remitimos a lo expuesto por (Filloy, Puig y Rojano, s.f) quienes aportan una herramienta vital para observar los problemas verbales que sobre el álgebra se construyen, en primer medida se debe conocer lo que es el lenguaje natural y el lenguaje algebraico, el primero da cuenta del lenguaje que para el niño es familiar y entiende, es decir pertenece a su contexto, este lenguaje deberá ser traducido al lenguaje algebraico, 
pero para ello el mensaje que se transmite en el problema debe ser claro, si esto no ocurre entonces el mensaje en lenguaje natural será abstracto y por ende su traducción al lenguaje algebraico también lo será, (Filloy et al, sf).

\section{Metodología.}

El desarrollo de esta investigación se ejecuta bajo las siguientes fases:

1. Revisión didáctica y matemática del concepto de producto notable y de representaciones semióticas.

2. Elección del libro que se analizará. Esta elección se hace basándose en una consulta sobre cuál fue el libro de matemáticas de grado 8 más vendido en el 2011 .

3. Elaboración de las categorías de análisis que son tomadas de (Beltran, 2012). Las cuales son:

a. Definición presentada para el concepto de producto notable

b. Ejemplos propuestos relacionados con el concepto de producto notable

c. Cómo presenta el texto el concepto de producto notable con respecto a los problemas y ejercicios

d. Significación operatoria de los registros de representación semiótica del producto notable.

4. Análisis en base a las categorías establecidas

5. Conclusiones.

\section{Análisis}

La representación semiótica que se privilegia para dar a entender la definición de este producto notable está dada en lenguaje natural, a continuación se evidencia en la imagen tal cual como aparece en el libro de texto.
Imagen 1

En general, el cuadrado de la suma de dos términos es igual al cuadrado del primer término más el doble del primer término por el segundo, más el cuadrado del segundo término Lenguaje Natural

Fuente: Elaboración propia

La definición general que se presenta al final, es resultado de una conversión que se realizó durante la presentación del producto notable, la cual se evidencia de la siguiente manera.

Ejemplos propuestos relacionados con el concepto de producto notable (cuadrado de la suma de dos términos):

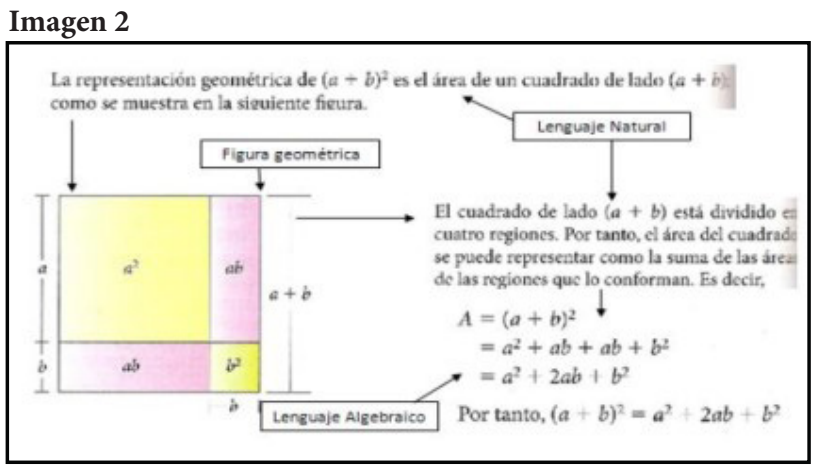

Fuente: Elaboración propia

Luego de la explicación de dicho producto notable, se presentan dos ejemplos los cuales pretenden dar por terminado éste, y poder pasar al siguiente, estos ejemplos son presentados al estudiante por medio de una representación semiótica de lenguaje algebraico y lenguaje natural, como lo muestra la siguiente imagen:

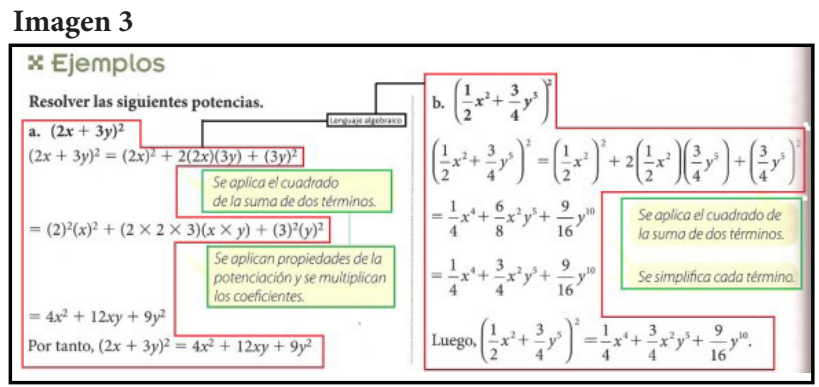

Fuente: Elaboración propia

En los recuadros rojos encontramos y evidenciamos representaciones en el lenguaje algebraico las cuales se privilegian más y en los recuadros verdes encontramos una representación auxiliar en lenguaje natural, ésta representación apoya el procedimiento que se está realizando para desarrollar la suma de de 
cuadrados de dos términos", la transformación que realiza el libro de texto para mostrar la solución en los ejemplos es de tratamiento ya que en los ejemplos se proponen dos expresiones a. $(2 x+3 y)^{2} \quad$ b. $\left(\frac{1}{2} x^{2}+\frac{3}{4} y^{5}\right)^{2}$ se hace un trabajo algebraico para llegar a la respuesta, en la transformación de tratamiento se produce respecto a un mismo objeto en este caso las expresiones a resolver. En los ejemplos las unidades significantes de cada representación semiótica que en este caso es lenguaje algebraico, son los símbolos, letras y números que hacen parte de las expresiones y desarrollo de las mismas según su signo operatorio.

Cómo presenta el texto el concepto de productos notables con respecto a los problemas y ejercicios.

Las transformaciones (tratamiento y conversión) que exige el problema o ejercicio. En las actividades propuestas por el texto, en la página 74 , se evidencia que la primera transformación que aparece es un tratamiento, puesto que se debe calcular el cuadrado de algunos monomios, por lo que tendrían que pasar a diferentes representaciones dentro del mismo registro, en este caso, el lenguaje algebraico. De este tipo aparecen en total 12 ejercicios.

A continuación se presenta una conversión, puesto que de la representación geométrica se pasa al lenguaje algebraico para saber cuál es el área de la figura. De esta transformación aparecen 4 ejercicios. Ejemplo:

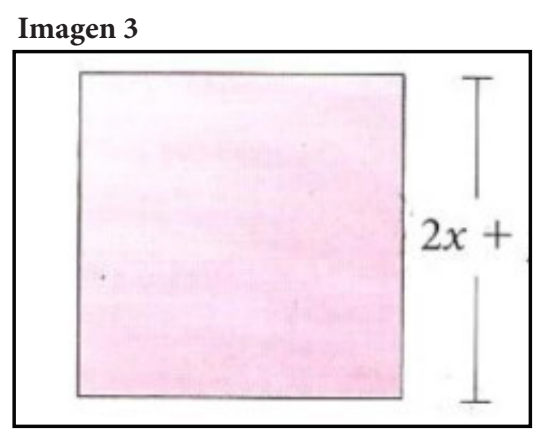

Fuente: Elaboración propia

Significación operatoria de los registros de representación semiótica de los productos notables. La primera transformación a la que se puede hacer referencia es el tratamiento pues en el ejercicio se pide al resolutor hallar las potencias del producto notable indicado, es decir que se moverá dentro de un mismo registro de representación presentando el mismo objeto $\{(6 x+1) 2\}$ de diferentes formas como se muestra a continuación.

Imagen 5

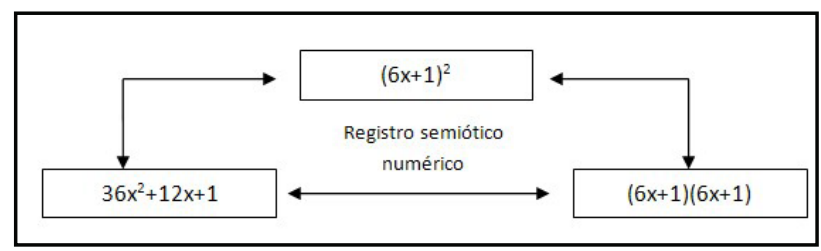

Fuente: Elaboración propia

Para el segundo caso (punto 7a) se puede observar que hay una conversión puesto que se manejan dos registros de representación que son el registro numérico y el geométrico que según (Duval, 2004). Están ubicados en los registros semióticos de lenguaje y visualización respectivamente. Se presenta entonces el paso de la representación geométrica a la representación numérica (cambio de registro).

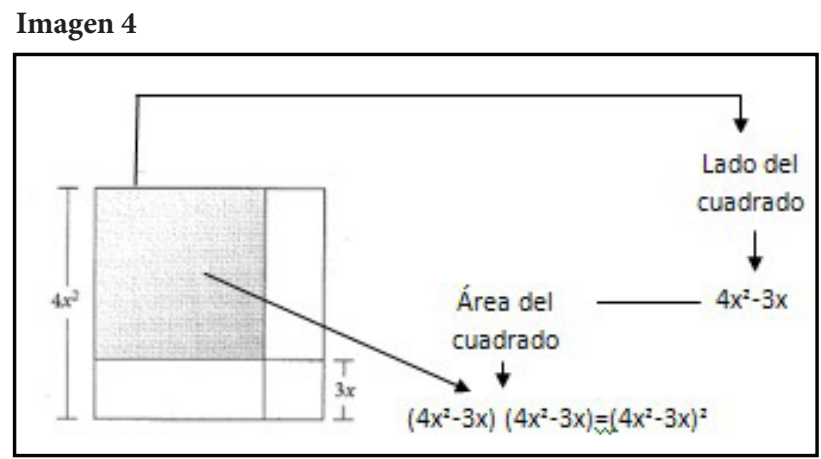

Fuente: Elaboración propia

\section{Conclusiones}

Gracias a este tipo de investigación que se hace sobre los aportes al álgebra a través de los libros de texto se debe observar con detenimiento el tipo de representación semiótica que se privilegia para establecer las relaciones que se pueden presentar entre el lenguaje natural y el lenguaje algebraico.

Se observa que dentro del libro de texto no se resalta la representación semiótica de la imagen, la presencia de este tipo de representación sería 
un factor importante en la adquisición del conocimiento pues entre más representaciones el niño pueda establecer sobre un mismo objeto mejor será su comprensión del mismo.

Se evidencia que en la unidad donde se presentan los productos notables se privilegian dos tipos de representaciones: lenguaje natural, algebraico y visualización, así mismo entre estas representaciones se producen transformaciones tanto de conversión como de tratamiento.

\section{Referencias}

Azcarate, P. Serrado, A. (2006) Tendencias didácticas en los libros de texto de matemáticas para la ESO. (pp341-378). Revista de educación, universidad de Cádiz.

Cervantes, M. (2010). Cómo enseñar productos notables. Recuperado de http://www.cienciaspuras.com/Datos/2\%20eso/COMO\%20 ENSENAR\%20LOS\%20PRODUCTOS\%20 NOTABLES.pdf
Duval, R., (2004) Semiosis y pensamiento humano, registros semióticos y aprendizajes intelectuales. Universidad del Valle. Editorial Peter Lang.

Kieran. C. (s.f). The Learning and Teaching of school Algebra.

Maldonado, M., Rodríguez, M., Tuyub, J. (2007). Un estudio sobre el discurso en los libros de texto de Matemáticas. Su relación con la práctica escolar (tesis). Universidad Autónoma de Yucatán.

Piñuel, J. (2002). Epistemología, metodología y técnicas del análisis de contenido. En Estudios de Sociolingüística (pp.1- 42). Madrid, España. Recuperado de http://www.ucm.es/ info/mdcs/A.Contenido.pdf 